\title{
The equivocal appendix at CT: prevalence in a control population
}

\author{
Emily M. Webb • Zhen J. Wang • Fergus V. Coakley • \\ Liina Poder • Antonio C. Westphalen • Benjamin M. Yeh
}

Received: 1 April 2009 / Accepted: 23 June 2009/Published online: 14 July 2009

(C) The Author(s) 2009. This article is published with open access at Springerlink.com

\begin{abstract}
The purpose of the study was to determine the prevalence of appendices with an equivocal appearance at computed tomography (CT) in a control population. We retrospectively identified a control population of 150 patients who underwent $\mathrm{CT}$ of the abdomen and pelvis for evaluation of hematuria (without abdominal pain, fever, or colonic disease). One reader measured the diameter of the appendix and noted if the appendix was either isodense in appearance or airless and fluid filled. Sixty-seven of 150 cases $(44.6 \%)$ demonstrated appendiceal diameter greater than $6 \mathrm{~mm}$. The appendix was collapsed or isodense in 34/ 150 cases $(22.7 \%)$. Only ten of 150 or $6.6 \%$ of cases were isodense in combination with diameter greater than $6 \mathrm{~mm}$, and none had diameter greater than $10 \mathrm{~mm}$. Only one of 150 cases $(0.67 \%)$ demonstrated airless fluid within the lumen, and the appendix measured less than $6 \mathrm{~mm}$. While the diameter of the normal appendix is frequently greater than $6 \mathrm{~mm}$, none measured greater than $10 \mathrm{~mm}$ in combination with ambiguous morphology. Furthermore, in the normal appendix, airless fluid filling the lumen is a rare appearance with a prevalence of less than $1 \%$. While appendicitis could undoubtedly occur in an isodense appendix between 6 and $10 \mathrm{~mm}$ in diameter, such an appearance can occur in up to $6.6 \%$ of the normal population
\end{abstract}

Keywords Normal appendix · Appendiceal diameter . Equivocal appendix $\cdot \mathrm{CT} \cdot$ Appendicitis

E. M. Webb $(\bowtie) \cdot$ Z. J. Wang $\cdot$ F. V. Coakley $\cdot$ L. Poder $\cdot$

A. C. Westphalen $\cdot$ B. M. Yeh

Department of Radiology, University of California, San Francisco,

M372, 505 Parnassus Avenue, P.O. Box 0628, San Francisco, CA 94143-0628, USA

e-mail: emma.webb@radiology.ucsf.edu

\section{Introduction}

Computed tomography (CT) has become the primary imaging modality for evaluation of adult patients with suspected appendicitis. While there are multiple signs of appendicitis at $\mathrm{CT}$, appendiceal enlargement is considered the most accurate with reported sensitivity and specificity of $92-93 \%$ and $92-100 \%$, respectively [1-3]. In most cases, patients with acute appendicitis also demonstrate secondary signs of inflammation at CT that aid in diagnosis. However, appendiceal enlargement is sometimes an isolated finding and has been reported to indicate acute appendicitis in up to $52 \%$ of symptomatic patients [4].

Therefore, knowledge of the parameters of normal appendiceal diameter is critical in suggesting or excluding a diagnosis of appendicitis at CT.

Early studies suggested that an appendix measuring greater than $6 \mathrm{~mm}$ was abnormal. This numeric threshold originated in the ultrasound literature [5-8] and was subsequently utilized in $\mathrm{CT}$ interpretation. However, the 6-mm threshold assumes a technique of graded compression of the appendix at ultrasound and is therefore difficult to extrapolate to $\mathrm{CT}$ interpretation. Prior studies have shown that in up to $42 \%$ of patients, the normal appendix measures greater than $6 \mathrm{~mm}$ in outer wall-to-outer wall diameter at CT [9, 10]. However, these studies have typically included appendiceal contents, such as air, in measurements of appendiceal diameter. In these instances, the fact that the appendix measures greater than $6 \mathrm{~mm}$ is of little clinical value, as a predominantly air-filled appendix lacking secondary signs of appendicitis would always be interpreted as normal at CT. Only normal appendices that are fluid filled or uniformly dense (where the luminal contents cannot be differentiated from the adjacent wall) might realistically be mistaken for appendicitis based on 
size criteria alone. Therefore, we undertook this retrospective study to determine the prevalence of morphologically equivocal, either fluid-filled or isodense appendices, measuring greater than $6 \mathrm{~mm}$ at $\mathrm{CT}$ in a control population.

\section{Materials and methods}

\section{Patients}

This single-institution study was approved by our Institutional Review Board and was compliant with requirements of the Health Insurance Portability and Accountability Act. The requirement for informed consent was waived. To determine the prevalence of fluid-filled or isodense appendices measuring greater than $6 \mathrm{~mm}$ at $\mathrm{CT}$, one author used a computer search to retrospectively identify all multidetector-row CT scans performed for evaluation of hematuria between January 2002 and January 2006. Only the first scan for each patient was included in the review. Electronic medical records were searched for relevant clinical history. Patients were excluded if they reported abdominal pain or fever at the time of imaging, had evidence of colonic wall thickening at CT, or if the appendix was not visualized. The scans of 150 consecutive patients were reviewed. The group included 96 women and 54 men with mean age of 54.7 (range 21 to 94 ).

\section{CT technique}

All 150 patients in the retrospective review group were scanned with multidetector-row CT (four, 16, or 64 detectors; HighSpeed or LightSpeed, General Electric, Milwaukee, WI, USA) using 1.25 or $5 \mathrm{~mm}$ slice thickness. Scans performed at $1.25 \mathrm{~mm}$ collimation were reconstructed to $5 \mathrm{~mm}$ slice thickness. The peak tube voltage was $120 \mathrm{kVp}$ and the milliampere was automatically adjusted to attain a noise index of 12. For 112 patients, $150 \mathrm{~mL}$ intravenous iohexol (Omnipaque 350 Nycomed Amersham, Princeton, NJ, USA) was injected at a rate of 3 to $5 \mathrm{~mL} / \mathrm{s}$, and the scan delay was $80 \mathrm{~s}$. Although these patients also had precontrast images performed, when postcontrast scans were available, they were preferentially used for retrospective review. Thirty-three of these patients also received $800 \mathrm{~mL}$ oral diatrizoate meglumine (Hypaque, Nycomed Amersham, Princeton, NJ, USA) prior to imaging. Routine administration of positive enteric contrast was only performed at our institution prior to 2006. Thirty-eight patients received neither oral nor intravenous contrast.

Image interpretation

One radiologist with 3 years subspecialty experience in abdominal imaging independently reviewed all CT images on a picture archiving and communication system workstation (IMPAX Version 4.5, Agfa, Mortsel, Belgium). In each case, the maximal outer wall-to-outer wall diameter of the appendix was measured to the nearest $0.5 \mathrm{~mm}$, and the average of three measurements was recorded. Measurements were obtained utilizing $200 \%$ magnification of axial images for all cases. The single wall thickness of the appendix was also measured and recorded in the same fashion. The predominant type of luminal contents (air, enteric contrast, or fluid) was recorded. An appendix was considered ambiguous in morphology if it had a uniformly collapsed, isodense appearance or contained airless, fluid within its lumen. Because it is difficult to differentiate a collapsed appendix from a distended appendix with contents of the same attenuation as the wall $[10,11]$, only the outer wall-toouter wall diameter was recorded when the luminal contents were not visually distinguishable from the wall.

\section{Statistical analysis}

Descriptive analysis was performed using the Stata software package version 8.0 (Stata Corporation, College Station, TX, USA) for Windows (Microsoft, Redmond, WA, USA). The Student $t$ test was used to compare the mean values of outer wall-to-outer wall appendiceal diameter between groups with different luminal contents and those with a collapsed or isodense appearance. In patients in whom the appendiceal wall was discernable, the Student $t$ test was used to compare the mean single wall thickness between groups with different luminal contents. Fisher's exact test was used to compare the proportions of patients with and without isodense appendices in the groups who received intravenous contrast or enteric contrast versus those who did not. A $p$ value $<0.05$ was considered to be statistically significant.

\section{Results}

The mean outer wall-to-outer wall diameter of the appendix for all 150 retrospectively evaluated CT scans was $6.3 \mathrm{~mm}$ (Table 1). Sixty-seven of 150 cases $(44.6 \%)$ had an outer wall-to-outer wall diameter greater than $6 \mathrm{~mm}$.

Of the $150 \mathrm{CT}$ scans, luminal contents were distinguishable from the appendiceal wall in 116 of 150 cases or $77 \%$. The mean outer wall-to-outer wall diameter and mean single wall thickness for this group are reported in Table 1 . The outer wall-to-outer wall diameter varied by type of luminal contents with the greatest mean diameter seen in those containing air, compared to those containing enteric contrast ( $p=0.01$ ) or fluid (Table 1; Figs. 1 and 2). Of note, only 12 of $33(36 \%)$ patients who were given positive oral contrast showed contrast filling the appendiceal lumen at 
Table 1 Mean outer wallto-outer wall diameter and single wall thickness of the appendix for 150 control patients

${ }^{\text {a }}$ Single wall thickness was only obtained in cases where the wall was visible separate from luminal contents

Mean appendix size

Maximal diameter (range) [mm]

Single wall thickness (range) [mm]

$\begin{array}{lll}\begin{array}{l}\text { CT appearance } \\ \text { All morphologies }(n=150)\end{array} & 6.3(3-10) & 1.6(0.5-3.5) \\ \text { Visible lumen contents } & & \\ \text { Overall }(n=116) & 6.3(4-9) & 1.6(0.5-3.5) \\ \text { Air in lumen }(n=103) & 6.4(4.5-9) & 1.6(0.5-3.5) \\ \text { Enteric contrast in lumen }(n=12) & 5.7(4-7) & 1.6(1-2) \\ \quad \text { Fluid in lumen }(n=1) & 6 & \mathrm{n} / \mathrm{a}^{\mathrm{a}} \\ \text { Isodense } & 5.7(3-10) & \\ \quad \text { Overall }(n=34) & & \end{array}$

CT. Only one case of $150(0.67 \%)$ had an ambiguous appearance due to airless fluid within the appendiceal lumen, and it measured less than $6 \mathrm{~mm}$ in outer wall-toouter wall diameter (Fig. 3). There was no statistical difference in single wall thickness of the appendix between groups with different luminal contents ( $p=0.2$ to 1.0 ).

Luminal contents were not discernable from the adjacent wall in 34 of 150 cases $(22.7 \%)$ resulting in an ambiguous morphology due to a collapsed or isodense appearance of the appendix (Fig. 4). Mean outer wall-to-outer wall diameter for this group is reported in Table 1 and is less than the mean diameter in cases with visible luminal contents $(p<0.01)$.

Only ten of 150 or $6.6 \%$ of appendices were isodense and had an outer wall-to-outer wall diameter greater than $6 \mathrm{~mm}$. None had diameter greater than $10 \mathrm{~mm}$. There was

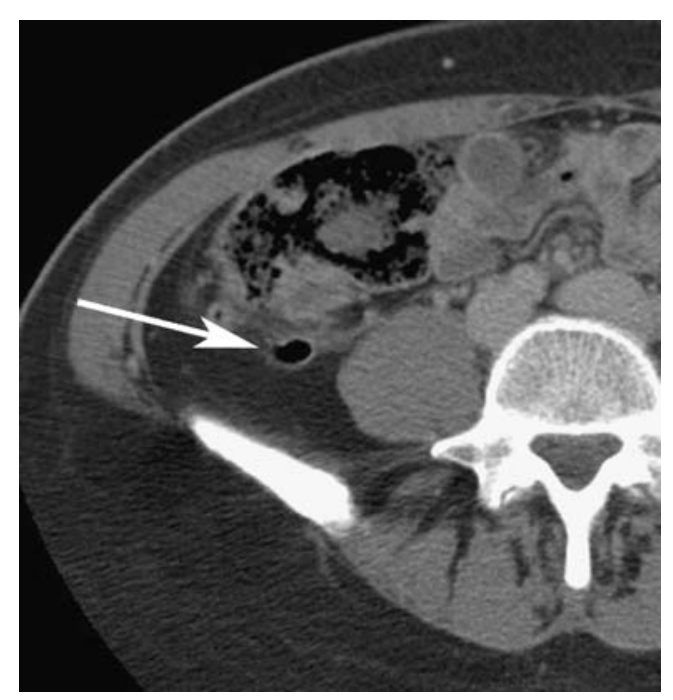

Fig. 1 Forty-two-year-old woman undergoing evaluation for hematuria. Noncontrast transverse CT image shows a normal air-filled appendix (arrow) measuring $9 \mathrm{~mm}$ in outer wall-to-outer wall diameter. Regardless of the diametric measurement, this appendix would always be interpreted as normal at CT based on the predominance of air within the appendiceal lumen no statistically significant difference between the proportion of isodense appendices seen on CT scans performed with ( 23 of 112 or $21 \%$ ) versus without intravenous contrast (11 of 38 scans or $29 \%, p=0.4)$. Furthermore, there was no statistically significant difference between the proportion of isodense appendices seen on CT scans performed with (12 of 33 or $36 \%$ ) versus without enteric contrast (22 of 117 or $19 \%, p>0.05)$. In fact, the appendix was slightly more likely to appear isodense when enteric contrast was administered.

\section{Discussion}

CT is the most common imaging modality used to evaluate adult patients with suspected appendicitis, with both high

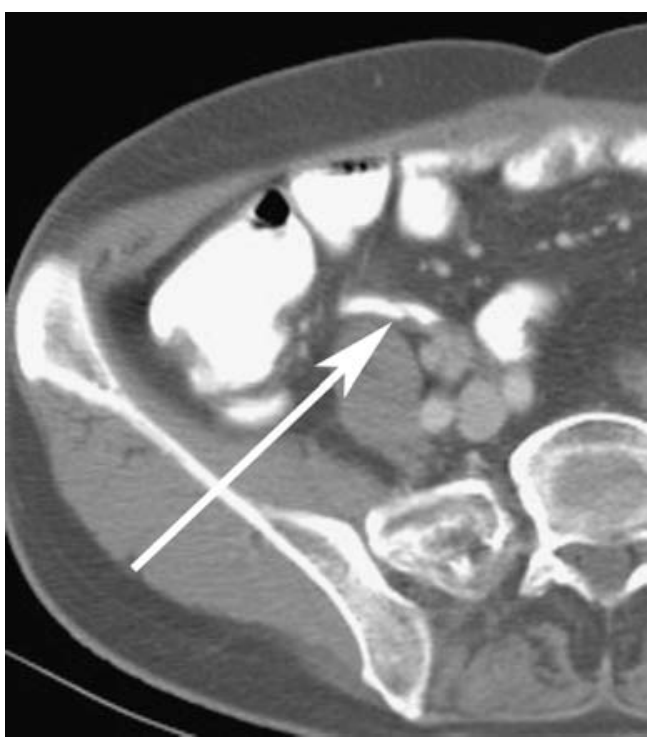

Fig. 2 Sixty-six-year-old man undergoing evaluation for hematuria. Contrast-enhanced transverse CT image shows a normal enteric contrast filled appendix (arrow) with outer wall-to-outer wall diameter of $7 \mathrm{~mm}$. Acute appendicitis can be excluded on the basis of morphology, regardless of maximal diameter 


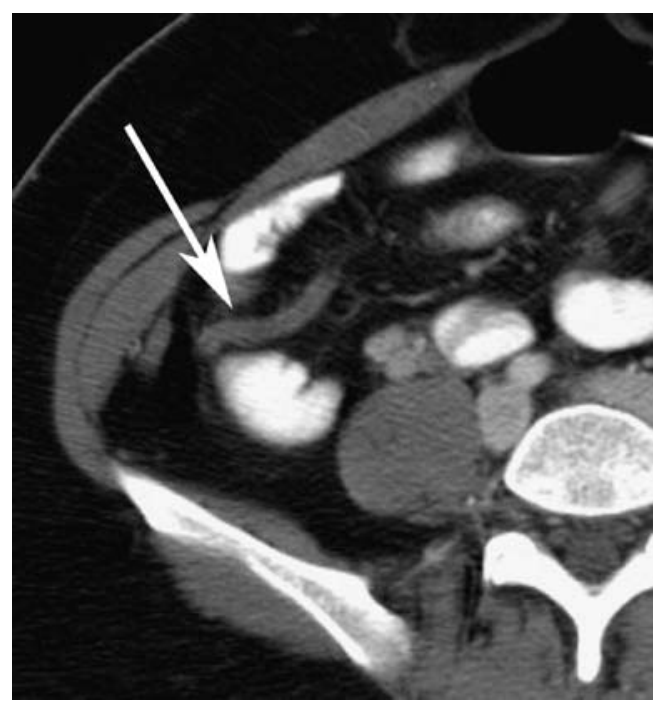

Fig. 3 Thirty-five-year-old woman undergoing evaluation for hematuria. Transverse CT image with intravenous and enteric contrast shows a normal appendix with a fluid-filled lumen (arrow). The outer wall-toouter wall diameter measures $6 \mathrm{~mm}$. The maximal depth of intraluminal fluid is $2 \mathrm{~mm}$. Airless fluid filling the appendiceal lumen is rare in an asymptomatic patient and should prompt a clinical evaluation for appendicitis

sensitivity and specificity for the diagnosis [12-22]. In most cases, CT interpretation is straightforward with cases definitively falling into either positive or negative categories. When appendicitis is present, we typically find both appendiceal enlargement and secondary signs of inflammation. In normal cases, by contrast, the appendix may demonstrate a range of sizes, but no secondary signs of inflammation are usually identified. Unfortunately, as appendiceal enlargement is sometimes the only sign of appendicitis, up to $12 \%$ of cases may be equivocal at CT [4].

As such, it is important for the radiologist to have a clear understanding of the size parameters of the normal appendix at CT. Similar to previous authors [9, 10], we found that $45 \%$ of normal appendices have outer wall-toouter wall diameter greater than $6 \mathrm{~mm}$ at CT. However, our study also indicates that in the majority of cases (77\%), the appendix had a recognizably normal morphology, either predominant luminal air or enteric contrast. Only $6.6 \%$ of normal appendices measured greater than $6 \mathrm{~mm}$ and also had a CT appearance that was ambiguous enough to be mistaken for appendicitis at CT, in other words, an isodense, collapsed appearance, or an airless fluid-filled lumen. None had diameter greater than $10 \mathrm{~mm}$ in combination with an ambiguous or equivocal morphology.

The pathophysiology of acute appendicitis usually involves appendiceal obstruction with continued mucinous fluid secretion and bacterial proliferation within the lumen of the appendix [23]. As such, a fluid- or mucus-filled appendix is recognized as a sign of acute appendicitis at CT [24]. To our knowledge, the incidence of this appearance in a normal population, however, has not been previously reported. In this study, only $0.67 \%$ or one of 150 of control patients demonstrated airless fluid in the appendiceal lumen. A recent study by Moteki and Horikoshi [24] found that depth of the intraluminal appendiceal fluid greater than $2.6 \mathrm{~mm}$ had high sensitivity and specificity $(>80 \%)$ for diagnosis of acute appendicitis. In our single normal appendix with a fluid-filled lumen, the maximal depth was only $2 \mathrm{~mm}$. In clinical practice, airless fluid is at least occasionally identified in the appendiceal lumen in patients with no clinical evidence for appendicitis, particularly in the setting of a fluid-filled cecum. However, given the rarity of this appearance in the normal appendix and its known association with acute appendicitis, the finding of a fluidfilled appendix with diameter greater than $6 \mathrm{~mm}$ should prompt a clinical work up for appendicitis (including blood work analysis and surgical evaluation), even in the absence of secondary signs of inflammation at CT. If the diagnosis remains unclear following clinical correlation, patient observation and short-interval follow-up CT may be appropriate.

Our study has several limitations. The primary limitation is the lack of a standard of reference for proof of a normal appendix. However, pathological correlation is unavailable in this population, as none of the patients included in our study had any clinical evidence of acute appendicitis or colonic disease. Furthermore, the outer wall-to-outer wall

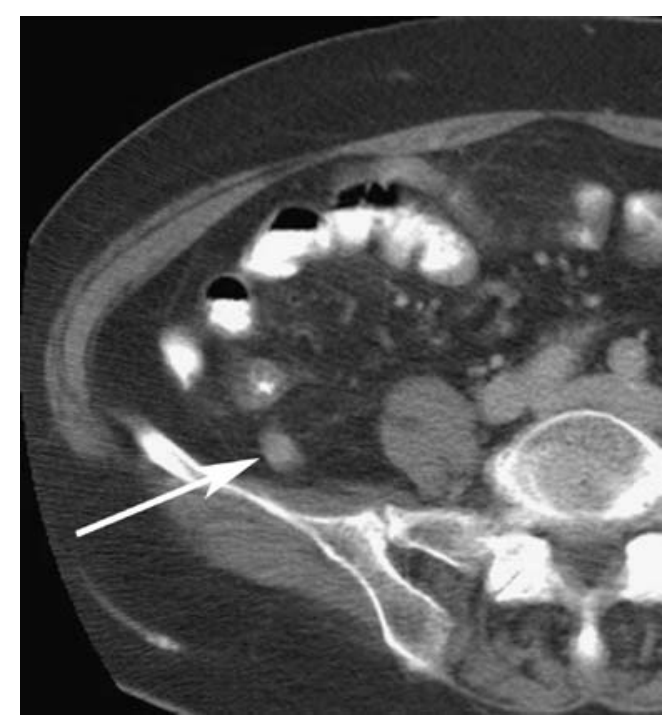

Fig. 4 Eighty-year-old woman undergoing evaluation for hematuria. Transverse CT image with intravenous and enteric contrast shows an isodense or collapsed appearing appendix in an asymptomatic patient measuring $1 \mathrm{~cm}$ in outer wall-to-outer wall diameter (arrow). A normal, but large, appendix with this morphology may be mistaken for early appendicitis at CT. In this case, it was queried on the radiologic report 
diameter of the appendix in our study is concordant with values previously reported in the $\mathrm{CT}$ literature for normal appendices [9-11]. Because the focus of our paper is on CT appearance of normal appendices, we also did not include a group of patients with clinically proven appendicitis to evaluate how many might have ambiguous CT findings of isodense or fluid-filled appendices. Another limitation is the retrospective nature of our study. Due to retrospective data collection, all patients were not scanned with intravenous contrast. It is possible that luminal contents may have been identified in more patients with an isodense-appearing appendix if intravenous contrast was administered to all patients. However, there was no statistical difference in the proportion of isodense appendices versus those with visible luminal contents between cases performed with versus without intravenous contrast.

In summary, our study found that while the outer wallto-outer wall diameter of the normal appendix is frequently greater than $6 \mathrm{~mm}$, none had diameter greater than $10 \mathrm{~mm}$ in combination with equivocal morphology. Furthermore, in the normal appendix, airless fluid within the lumen is rarely seen with a prevalence of less than $1 \%$. Therefore, a fluidfilled appendix should alert radiologists and clinicians for the possibility of appendicitis, even in the absence of secondary signs of inflammation at CT. While appendicitis could undoubtedly occur in an isodense appendix measuring between 6 and $10 \mathrm{~mm}$ in diameter, such an equivocal appearance can occur in up to $6.6 \%$ of the normal population.

Open Access This article is distributed under the terms of the Creative Commons Attribution Noncommercial License which permits any noncommercial use, distribution, and reproduction in any medium, provided the original author(s) and source are credited.

\section{References}

1. Rao PM, Rhea JT, Novelline RA (1997) Sensitivity and specificity of the individual CT signs of appendicitis: experience with 200 helical appendiceal CT examinations. J Comput Assist Tomogr 21 (5):686-692

2. Choi D, Park H, Lee YR, Kook SH, Kim SK, Kwag HJ, Chung EC (2003) The most useful findings for diagnosing acute appendicitis on contrast-enhanced helical CT. Acta Radiol 44 (6):574-582

3. Ives EP, Sung S, McCue P, Durrani H, Halpern EJ (2008) Independent predictors of acute appendicitis on CT with pathologic correlation. Acad Radiol 15(8):996-1003

4. Daly CP, Cohan RH, Francis IR, Caoili EM, Ellis JH, Nan B (2005) Incidence of acute appendicitis in patients with equivocal CT findings. AJR Am J Roentgenol 184(6):1813-1820

5. Jeffrey RB Jr, Laing FC, Townsend RR (1988) Acute appendicitis: sonographic criteria based on 250 cases. Radiology 167(2):327-329
6. Rioux M (1992) Sonographic detection of the normal and abnormal appendix. AJR Am J Roentgenol 158(4):773-778

7. Simonovský V (1999) Sonographic detection of normal and abnormal appendix. Clin Radiol 54(8):533-539

8. Vignault F, Filiatrault D, Brandt ML, Garel L, Grignon A, Ouimet A (1990) Acute appendicitis in children: evaluation with US. Radiology 176(2):501-504

9. Tamburrini S, Brunetti A, Brown M, Sirlin CB, Casola G (2005) CT appearance of the normal appendix in adults. Eur J Radiol 15 (10):2096-2103

10. Benjaminov O, Atri M, Hamilton P, Rappaport D (2002) Frequency of visualization and thickness of normal appendix at nonenhanced helical CT. Radiology 225(2):400-406

11. Bursali A, Arac M, Oner AY, Celik H, Eksioglu S, Gumus T (2005) Evaluation of the normal appendix at low-dose nonenhanced spiral CT. Diagn Interv Radiol 11(1):45-50

12. Balthazar EJ, Megibow AJ, Siegel SE, Birnbaum BA (1991) Appendicitis: prospective evaluation with high-resolution CT. Radiology 180(1):21-24

13. Malone AJ Jr, Wolf CR, Malmed AS, Melliere BF (1993) Diagnosis of acute appendicitis: value of unenhanced CT. AJR Am J Roentgenol 160(4):763-766

14. Rao PM, Rhea JT, Novelline RA, Mostafavi AA, Lawrason JN, McCabe CJ (1997) Helical CT combined with contrast material administered only through the colon for imaging of suspected appendicitis. AJR Am J Roentgenol 169(5):1275-1280

15. Rao PM, Rhea JT, Novelline RA et al (1997) Helical CT technique for the diagnosis of appendicitis: prospective evaluation of a focused appendix CT examination. Radiology 202(1):139 144

16. Funaki B, Grosskreutz SR, Funaki CN (1998) Using unenhanced helical CT with enteric contrast material for suspected appendicitis in patients treated at a community hospital. AJR Am J Roentgenol 171(4):997-1001

17. Lane MJ, Liu DM, Huynh MD, Jeffrey RB Jr, Mindelzun RE, Katz DS (1999) Suspected acute appendicitis: nonenhanced helical CT in 300 consecutive patients. Radiology 213(2):341346

18. Stroman DL, Bayouth CV, Kuhn JA et al (1999) The role of computed tomography in the diagnosis of acute appendicitis. Am J Surg 178(6):485-489

19. Kamel IR, Goldberg SN, Keogan MT, Rosen MP, Raptopoulos V (2000) Right lower quadrant pain and suspected appendicitis: nonfocused appendiceal CT-review of 100 cases. Radiology 217 (1):159-163

20. Jacobs JE, Birnbaum BA, Macari M et al (2001) Acute appendicitis: comparison of helical CT diagnosis focused technique with oral contrast material versus nonfocused technique with oral and intravenous contrast material. Radiology 220 (3):683-690

21. Wise SW, Labuski MR, Kasales CJ et al (2001) Comparative assessment of CT and sonographic techniques for appendiceal imaging. AJR Am J Roentgenol 176(4):933-941

22. Raman SS, Lu DS, Kadell BM, Vodopich DJ, Sayre J, Cryer H (2002) Accuracy of nonfocused helical CT for the diagnosis of acute appendicitis: a 5-year review. AJR Am J Roentgenol 178 (6):1319-1325

23. Crawford JM (1994) The gastrointestinal tract. In: Cotran RS, Kumar V, Robbins SL (eds) Robbins pathologic basis of disease. Saunders, Philadelphia, p 823

24. Moteki T, Horikoshi H (2007) New CT criterion for acute appendicitis: maximum depth of intraluminal appendiceal fluid. AJR Am J Roentgenol 188(5):1313-1319 